\title{
In vivo visualization of cells labeled with superparamagnetic iron oxides by a sub-millisecond gradient echo sequence
}

\author{
Xeni Deligianni · Daniel Jirák · Zuzana Berková • \\ Milan Hájek · Klaus Scheffler · Oliver Bieri
}

Received: 8 March 2013/Revised: 30 October 2013/Accepted: 7 November 2013/Published online: 30 November 2013 (c) ESMRMB 2013

\begin{abstract}
Object In vivo magnetic resonance imaging (MRI) of iron-labeled pancreatic islets (PIs) transplanted into the liver is still challenging in humans. The aim of this study was to develop and evaluate a double contrast method for the detection of PIs labeled with superparamagnetic iron oxide (SPIO) nanoparticles.

Materials and methods A double-echo three-dimensional (3D) spoiled gradient echo sequence was adapted to yield a sub-millisecond first echo time using variable echo times and highly asymmetric Cartesian readout. Positive contrast was achieved by conventional and relative image subtraction. Experiments for cell detection efficiency were
\end{abstract}

Xeni Deligianni and Daniel Jirák have contributed equally to this work.

X. Deligianni $(\bowtie) \cdot$ O. Bieri

Division of Radiological Physics, Department of Radiology,

Clinic of Radiology and Nuclear Medicine, University of Basel

Hospital, Petersgraben 4, 4031 Basel, Switzerland

e-mail: xeni.deligianni@unibas.ch

\section{Deligianni · K. Scheffler}

High-Field Magnetic Resonance Center, Max-Planck Institute for Biological Cybernetics, Tübingen, Germany

D. Jirák · Z. Berková · M. Hájek

Institute for Clinical and Experimental Medicine, Prague,

Czech Republic

D. Jirák

Institute of Biophysics and Informatics, 1st Medicine Faculty, Charles University, Prague, Czech Republic

K. Scheffler

Department of Biomedical Magnetic Resonance, University

Hospital Tübingen, Tübingen, Germany performed in vitro on gelatin phantoms, in vivo on a Lewis rat and on a patient 6 months after PI transplantation.

Results It was demonstrated that the proposed method can be used for the detection of transplanted PIs with positive contrast in vitro and in vivo. For all experiments, relative subtraction yielded comparable and in some cases better contrast than conventional subtraction. For the first time, positive contrast imaging of transplanted human PIs was performed in vivo in patients.

Conclusion The proposed method allows 3D data acquisition within a single breath-hold and yields enhanced contrast-to-noise ratios of transplanted SPIO labeled pancreatic islets relative to negative contrast images, therefore providing improved identification.

Keywords Variable echo time $\cdot 3 \mathrm{~T}$ MRI $\cdot$ SPIO · Pancreatic islets $\cdot$ Diabetes $\cdot$ Positive contrast

\section{Introduction}

Superparamagnetic iron oxide nanoparticles (SPIO) induce strong susceptibility effects that lead to a pronounced shortening of both $\mathrm{T}_{2}$ and $\mathrm{T}_{2}{ }^{*}$ relaxation times. Single cells are not visible with magnetic resonance imaging (MRI), but can be traced upon labeling with SPIO [1-3]. One of the most successful applications of cell imaging is the monitoring of transplanted pancreatic islets (PIs). Transplantation of PIs into the liver represents a promising alternative treatment to the well-established pancreas organ transplantation for restoring normoglycemia in type 1 diabetes mellitus patients [4, 5]. In 2004, Jirak et al. [6] demonstrated that SPIO-labeled pancreatic islets can be detected as hypointense spots on $\mathrm{T}_{2}{ }^{*}$ images following transplantation into the rat liver. Since then, many other 
studies have been performed to monitor the fate of labeled PIs with MRI on animal models [7-9]. Recently, Jiao et al. [8] confirmed that ex vivo cell labeling for islet graft imaging and monitoring with a $3 \mathrm{~T}$ clinical scanner is feasible and advantageous over $1.5 \mathrm{~T}$, since the susceptibility effect of iron nanoparticles is amplified.

Noninvasive islet graft MR-imaging is successfully applied in human livers up to 6 months after transplantation, despite signal loss due to the clearance of the labeling from the islets [7, 10-12]. Although the signal loss is clearly visible and can be monitored, quantitative or serial studies are not easily performed [10, 13, 14]. Typically, the iron-labeled islets appear as hypointense spots on conventional $\mathrm{T}_{2}$ or $\mathrm{T}_{2}{ }^{*}$ MRI, but susceptibility artifacts, such as air-tissue interfaces, or other effects like calcification or flow-related signal loss, could also yield negative contrast, and thus reliable identification of false positives can become an issue. In addition, using the signal void as a means of detection can be hindered by severe partial volume effects [14]. As a result, positive contrast MR techniques [15-18] such as double echo ultrashort echo time (UTE) methods were proposed to prevent such false positive results [19]. Recently, the feasibility of quantitative imaging and automatic thresholding of islets labeled with iron oxides with a positive contrast UTE-based MRI method was shown [19-21]. UTE allows successful islet detection in rats, but image acquisition requires respiratory triggering, which in this case was achieved with a specialized respiratory pad system [19]. In humans, however, UTE with respiratory triggering for abdominal imaging has not yet been applied.

In UTE techniques, it is common to use a subtraction image (i.e., subtracting a later echo from the UTE image) in order to yield positive contrast and increase the conspicuity in the detection of labeled islets $[14,21]$. This kind of positive contrast serves in reducing false positives, but in principle, the two images could also be used for weighted subtraction in order to yield a weighted positive contrast. In 2013, Martirosian et al. [22] proposed a relative subtraction method to enhance the positive contrast in a modified DESS sequence.

Contemporary imaging protocols used in standard clinical practice for the follow-up of the islet grafts in patients include balanced steady-state free precession (bSSFP) and spoiled gradient echo (SPGR) sequences [11]. Gradient echo methods provide good anatomic liver images, but usually only a few two-dimensional (2D) slices can be acquired in a breath-hold. The goal of this study was to develop an MRI technique for $3 \mathrm{~T}$ scanners that could monitor the fate of transplanted labeled islets in patients. As a result, we modified a contemporary sequence that was already being used in the clinic for the screening of diabetic patients after transplantation. Instead of the typically implemented 2D protocol, we used a three-dimensional (3D) protocol to achieve larger volume coverage with improved signal-to-noise ratio. A Cartesian sub-millisecond echo time SPGR 3D sequence was used and optimized for the in vivo visualization of SPIOlabeled PIs in the liver, in order to yield positive contrast images from patient single-breath-hold imaging. Visualization of pancreatic islets was explored with conventional and weighted subtraction images. The variable echo time scheme in combination with a highly asymmetric readout, as demonstrated in a previous study [23], allowed an important reduction of the echo time down to $0.65 \mathrm{~ms}$, while simultaneously achieving a sufficiently high resolution (i.e., $1.3 \times 1.3 \mathrm{~mm}^{2}$ in-plane) for in vivo scanning of humans.

\section{Materials and methods}

\section{Animal experiments}

All protocols were approved by the Ethics Committee of the Institute for Clinical and Experimental Medicine and the Committee for Animal Care of the Second Faculty of Medicine of Charles University. The experiments were carried out in accordance with the European Communities Council Directive of 24 November 1986 (86/609/ $\mathrm{EEC})$.

Islet isolation, labeling and transplantation for animal experiments

Male Lewis rats (Anlab, Czech Republic) were used as islet donors, as well as recipients for in vivo experiments. The pancreases of donor rats were distended by collagenase (1 mg/ml; Sevapharma, Czech Republic) and incubated at $37{ }^{\circ} \mathrm{C}$ in a water bath for $20 \mathrm{~min}$. The islets were separated from exocrine tissue by centrifugation in a discontinuous Ficoll gradient (Sigma, USA). Purified islets were cultivated in CMRL-1066 medium (PANBiotech GmbH, Germany) supplemented with $10 \%$ fetal calf serum, $1 \%$ penicillin/streptomycin/L-glutamine and $1 \%$ HEPES (all reagents from Sigma, USA) in a humidified $\mathrm{CO}_{2}$ incubator at $37{ }^{\circ} \mathrm{C}$ and $5 \% \mathrm{CO}_{2}$ atmosphere. For islet labeling, the MR contrast agent ferucarbotran (RESOVIST ${ }^{\circledR}$; Schering AG, Germany) was used $(5 \mu \mathrm{l} / \mathrm{ml})$ and pancreatic islets were cultured for $24 \mathrm{~h}$. Before transplantation, labeled islets were washed three times in Hanks Balanced Saline Solution (Sigma, USA). A total of 1,500 hand-picked labeled islets were transplanted under general anesthesia into the portal veins of recipients.

Human islet isolation, labeling and transplantation

After purification and washing, the islets were transferred into the culture medium CMRL-1066 (PAN Biotech 
GmbH, Germany), which was modified and supplemented according to the Miami Center [23]. Before culture, MRI contrast agent ferucarbotran $5 \mu \mathrm{L} / \mathrm{mL} \quad\left(\right.$ RESOVIST $^{\circledR}$; Schering AG, Germany) was added. The islets were in culture for $24 \mathrm{~h}$, and before transplantation, the labeled islets were washed again in the culture medium to remove the free ferucarbotran. Then 320,000 islet equivalents were infused in local anesthesia percutaneously under radiologic control into the portal vein within approximately $60 \mathrm{~min}$.

\section{MRI imaging}

\section{Sub-millisecond SPGR sequence}

A double contrast selective 3D SPGR sequence with a variable first echo time (TE) in combination with a highly asymmetric readout was used [23]. Here, the TE of the first echo varies dynamically across $\mathrm{k}$-space, enabling a minimum effective echo time, while highly asymmetric sampling allows for even further reduction of the echo time. Both echoes were acquired after each single excitation. This provided sub-millisecond TE for the first echo, as a key for positive contrast imaging and monitoring of islets labeled with SPIO. Furthermore, islet detection is facilitated within a stack of contiguous slices that is a $3 \mathrm{D}$ acquisition. A 3D sequence enables easier acquisition of multiple contiguous slices (i.e., in respect to 2D) to facilitate the islet detection. A special projection onto convex sets algorithm [24] provided by the manufacturer was used to compensate for the asymmetric data sampling.

Positive contrast and relative subtraction images

In order to yield positive contrast, a later echo was subtracted from the sub-millisecond first echo, and the image difference was reconstructed online [25]. Apart from the subtraction images, weighted subtraction images were also produced in post-processing. As has been demonstrated by Martirosian et al. [22], by adding a weighting factor, the positive contrast can appear significantly higher. Relative subtraction images were reconstructed offline with Matlab 7.0 (The Mathworks, Inc., MA, USA, R2008b) according to the following formula:

$\left(\mathrm{S}_{\mathrm{TE}_{1}}-\mathrm{S}_{\mathrm{TE}_{2}}\right) /\left(\mathrm{S}_{\mathrm{TE}_{1}}+\mathrm{S}_{\mathrm{TE}_{2}}\right)$

where $\mathrm{S}_{\mathrm{TE}_{1}}$ is the signal from the first short echo and $\mathrm{S}_{\mathrm{TE}_{2}}$ the signal from the later echo.

\section{Contrast analysis}

In order to yield a quantitative estimation of the quality of the contrast, relative contrast-to-noise ratios (CNR) were calculated. For the evaluation of the islet-related signal loss in the first and second echo images, contrast ratios were calculated according to the following equation:

$\mathrm{CNR}_{A}=\frac{\mathrm{SNR}_{\mathrm{BG}}-\mathrm{SNR}_{\mathrm{ROI}}}{\mathrm{SNR}_{\mathrm{BG}}}$

where $S N R_{R O I}$ is the signal-to-noise ratio (i.e., mean value of the signal divided by the standard deviation of the noise) of the labeled islets, and $S N R_{B G}$ is the SNR of the signal in a ROI in the islet surroundings (i.e., the liver tissue in the animal and human scans). For the evaluation of the positive islet contrast in the image difference and relative subtraction image, contrast ratios were calculated from:

$\mathrm{CNR}_{B}=\frac{\mathrm{SNR}_{\mathrm{ROI}}-\mathrm{SNR}_{\mathrm{BG}}}{\mathrm{SNR}_{\mathrm{ROI}}}$

\section{Phantom imaging}

Pancreatic rat and human islets were labeled with Resovist (Schering AG, Germany) for 1 day, and were then inserted into a cylindrical gel phantom in order to test the sequence performance with respect to islet separation and detection. Human and rat islets were separated in two different small tubes and then placed in a larger cylindrical phantom filled with gel. Labeled islets were placed in the surrounding gel as well. Phantom scans were performed in a whole body $3 \mathrm{~T}$ clinical system (Verio, Siemens Erlangen). For protocol details, see Table 1.

\section{Animal MR imaging}

The Lewis rat was scanned in a whole body clinical system (3T Tim Trio, Siemens Medical Solutions, Erlangen, Germany) with a dedicated 8-channel transmit/receive animal coil (Rapid Biomedical, Germany). For protocol details, see Table 1. For reference, high-resolution (0.23 mm in-plane) standard SPGR images ( $\mathrm{TE}=3.7 \mathrm{~ms})$ were acquired using a 4.7T Bruker Biospec Spectrometer equipped with a resonator coil (Bruker, Germany). During the examination, anesthesia was induced and continued by spontaneous breathing of $3 \%$ and 1-2\% isoflurane in the surrounding air, at $3 \mathrm{~T}$ and $4.7 \mathrm{~T}$ respectively.

\section{Human imaging}

The protocol was tested and evaluated on three healthy volunteers, and subsequently a patient, who was a member of the cohort of patients studied under the ENCITE project [www.encite.org], was scanned 6 months after transplantation [9] on a 3T whole body clinical scanner (Tim Trio, Siemens Medical Solutions, Erlangen, Germany). The study was approved by the Ethics Committee of the Institute for Clinical and Experimental Medicine. All participants of the project were informed about the nature and potential risks 
Table 1 Protocol parameters used at 3T

\begin{tabular}{|c|c|c|c|c|c|}
\hline \multirow[t]{2}{*}{ 3T MRI } & \multirow[t]{2}{*}{ Phantom } & \multirow{2}{*}{$\begin{array}{c}\text { Rat scans } \\
\text { 3D sub-ms SPGR }\end{array}$} & \multicolumn{3}{|l|}{ Patient scan } \\
\hline & & & & 2D axial SPGR & bSSFP (three phase cycles) \\
\hline In-plane resolution (mm) & 1.25 & 0.74 & 1.33 & 0.8 & 1.3 \\
\hline FOV $(\mathrm{mm})$ & $400 \times 275$ & $380 \times 261$ & $340 \times 244$ & $400 \times 262$ & $400 \times 250$ \\
\hline Slices/slice thickness (mm) & $10 / 2.5$ & $16 / 1.25$ & $8 / 2.5$ & $2 / 2.5$ & $12 / 2.5$ \\
\hline Bandwidth (Hz/pixel) & 401 & 515 & 455 & 315 & 745 \\
\hline $\mathrm{TE}_{1}(\mathrm{~ms}) / \mathrm{RO}$ asymmetry $(\%)^{\mathrm{a}}$ & $0.61 / 9$ & $0.8 / 15.6$ & $0.65 / 19.5$ & $5 / 100$ & $2 / 100$ \\
\hline $\mathrm{TE}_{2}(\mathrm{~ms}) / \mathrm{RO}$ asymmetry $(\%)$ & $6 / 100$ & $6 / 100$ & $6 / 100$ & - & - \\
\hline TR (ms)/Flip angle $\left(^{\circ}\right)$ & $15 / 8$ & $15 / 8$ & $10 / 8$ & $20 / 30$ & $4 / 40-60$ \\
\hline Acquisition time & $23.11 \mathrm{~s}$ & $8.28 \mathrm{~min}$ & $19 \mathrm{~s}$ & $18 \mathrm{~s}$ & $20 \mathrm{~s}$ \\
\hline Number of signal averages & 1 & 4 & 2 & 4 & 1 \\
\hline Slice oversampling $(\%)$ & 60 & 50 & 50 & - & - \\
\hline
\end{tabular}

${ }^{a}$ RO asymmetry (Readout asymmetry): percentage of readout sampling before the center of k-space

of the study and signed their informed consent. The subjects were examined in supine position with a 6-channel body phased-array coil placed on the upper abdomen.

Besides the 3D variable echo time (vTE) SPGR scan (see Table 1), the examination included a typical clinical protocol setting, comprising an axial and coronal HASTE for anatomical reference and a balanced SSFP sequence, as well as a current 2D SPGR protocol for pancreatic islet detection (cf [11]; for comparison of protocol parameters, see Table 1), and the whole examination did not exceed $1 \mathrm{~h}$. The additional positive contrast scans increased the total scan protocol by three breath-hold scans of $19 \mathrm{~s}$ each (i.e., the acquisition was repeated to scan different parts of the liver); a 3D transverse data set with $50 \%$ slice oversampling was acquired in a single breath-hold at end-expiration mode. GRAPPA acceleration of a factor of 2 and Partial Fourier 6/8 in slice direction was used to reduce the scan time. The flip angle was adjusted in order to yield optimum signal from the liver so that the long $\mathrm{T}_{2}$ species suppression would be optimal. For protocol details, see Table 1.

\section{Results}

\section{Phantom scan}

The sub-millisecond short echo time image (see Fig. 1a) exhibited homogenous signal, and almost no hypointensities were present. In the second echo (see Fig. 1b), islets appeared as dark areas of smaller or bigger extent, as a result of the different amount of contrast agent. In the image difference (see Fig. 1c) and in the relative image difference (see Fig. 1d), islets appeared as hyperintense areas that could easily be distinguished from the background. The contrast of the islets looks similar in the image difference and in the relative image difference. Both images yielded better CNR in comparison to the late echo, while the CNRs with both positive contrast methods were almost identical (see Table 2). The smallest visible hyperintensities could be attributed to single islets, which was verified by visual observation.

\section{Animal scan}

In the animal subject, the liver exhibited completely homogenous signal in the sub-millisecond first echo image acquired with the proposed method (see Fig. 2ai), while the labeled islets appeared as dark spots in the second echo (see Fig. 2aii). The short echo image provided enough signal in the proximity of the islets for the reconstruction of a positive contrast image (see Fig. 2aiii), from which the islets appeared hyperintense and could easily be identified. On the relative subtraction images (see Fig. 2aiv), equal amounts of hyperintense spots were detected, while the contrast-to-noise was improved (see Table 2). As can also be observed in the zoomed images (see Fig. 2b), the relative-subtraction images yielded better contrast for the islets.

The location and presence of islets was confirmed by comparison to the high-resolution images from the experimental 4.7T scanner. The same position was confirmed by looking at bigger anatomical structures. On Fig. 2c, a highresolution image from the experimental scanner is presented, which corresponds to the same position as the vTE SPGR images (see Fig. 2a). By comparison of the respective images, it was possible to confirm that the contrast observed on the positive contrast images was indeed due to the presence of labeled islets.

\section{Patient scan}

Transplanted pancreatic islets could be identified in $\mathrm{T}_{2}$-weighted and in positive contrast images in only a few positions (i.e., positive contrast images were acquired only in selected positions). In patient positive contrast images, 
Fig. 1 Cylindrical gel phantom with rat and islets. The circular forms visible in the short $\mathbf{a}$ and long, $\mathbf{b}$ echo image are smaller gel containers to separate the rat from the human islets. The images were acquired at $3 \mathrm{~T}$ field strength: a sub-millisecond first echo image acquired at $0.61 \mathrm{~ms}$, b second echo image acquired at $6 \mathrm{~ms}$, c positive contrast image (subtraction of the second echo from the first echo), where the islets appear as hyperintensities, d relative subtraction image.

The ROIs for which the CNRs were calculated are indicated on sub-Fig. $\mathbf{b}$
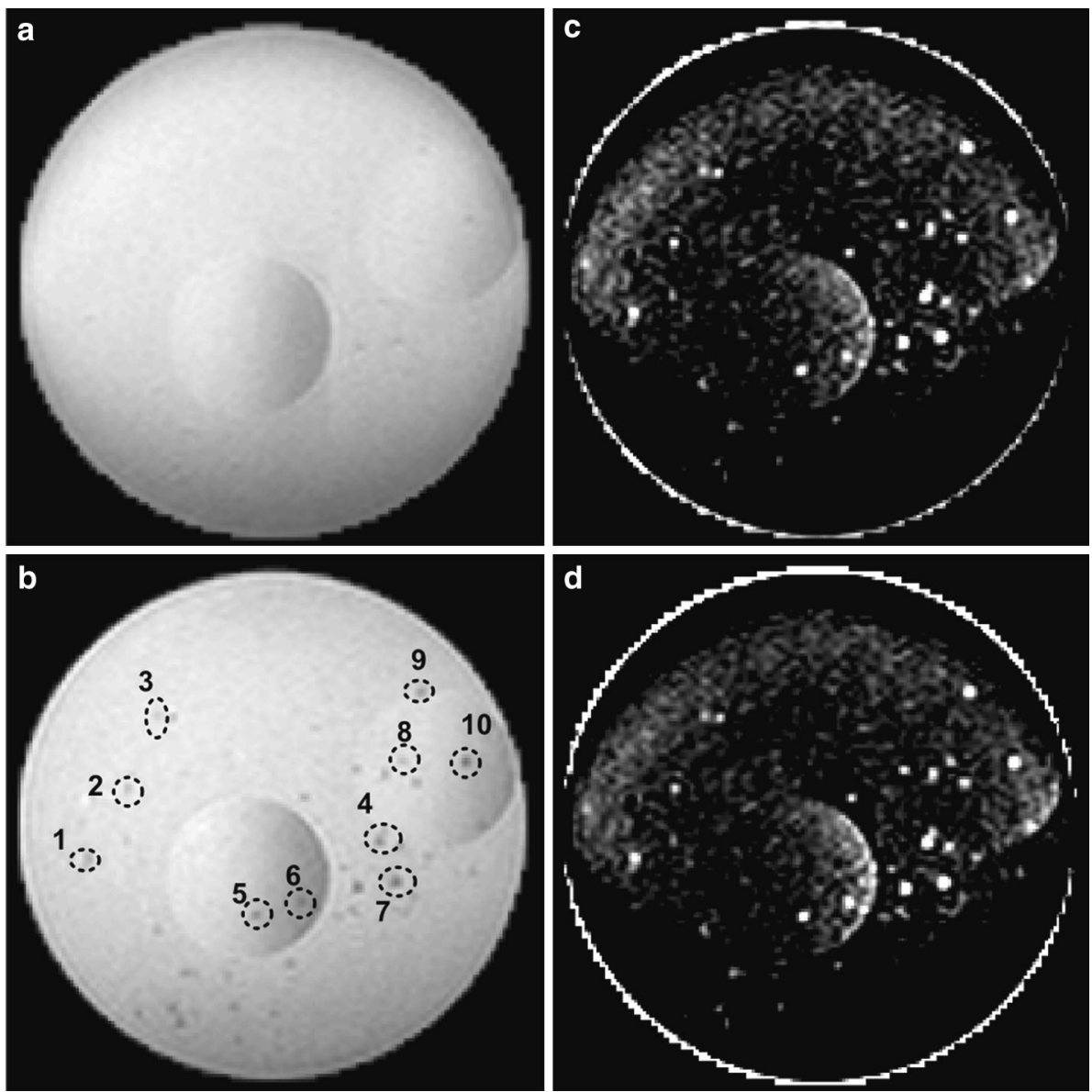

Table 2 CNRs for selected ROIs from Figs. 1, 2 and 3

\begin{tabular}{lllllll}
\hline & & \multicolumn{2}{l}{$\mathrm{CNR}_{\mathrm{A}}$} & & \multicolumn{2}{l}{$\mathrm{CNR}_{\mathrm{B}}$} \\
& & $\mathrm{TE}_{1}$ & $\mathrm{TE}_{2}$ & & Pos. Contrast & Rel. Subtraction \\
\hline Phantom & $\mathrm{ROI}_{1-10}$ & $0.01 \pm 0.03$ & $0.11 \pm 0.04$ & & $0.87 \pm 0.08$ & $0.88 \pm 0.08$ \\
Rat & $\mathrm{ROI}_{1-5}$ & $0.05 \pm 0.03$ & $0.27 \pm 0.05$ & & $0.26 \pm 0.05$ & $0.44 \pm 0.10$ \\
Patient & $\mathrm{ROI}_{1}$ & 0.00 & 0.15 & 0.37 & 0.43 \\
& $\mathrm{ROI}_{2}$ & 0.03 & 0.31 & 0.64 & 0.71 \\
\hline
\end{tabular}

The ROIs are indicated on the figures of the relevant experiments. The standard deviation is calculated out of the mean value of all ten or five ROIs for the phantom and rat images, respectively. Positive contrast images have higher contrast than the second echo images, and the relative subtraction images have comparable or higher ratios in comparison to positive contrast images

islets were detected on two different non-adjacent slices (see Fig. 3 left and right). They could be identified as regions without any hypointensities present in the submillisecond first echo acquired at $0.65 \mathrm{~ms}$ (see Fig. 3a) and hypointense regions on the second echo (see Fig. 3b). On the positive contrast image (i.e., the image difference), distributed hyperintensities (see Fig. 3c) were visible in the close vicinity of the islets. On the relative subtraction images, islets were also identified in both positions (see Fig. 3d). The relative subtraction images offer better background suppression, and therefore better contrast-to- noise (see Table 2). In one of the slices (see Fig. 3c right), positive contrast imaging revealed the possible presence of an islet not discernible on the later echo image. From its overall characteristics of appearance, i.e. its location, signal amplitude and shape, the hyperintensity most likely reflects the presence of a labeled pancreatic islet. The fact that with the positive contrast scans islets were identified only in two locations could be attributed to the fact that the patient was scanned 6 months after transplantation where the majority of the iron was cleared out from islets or islets were already lost $[6,11]$. 

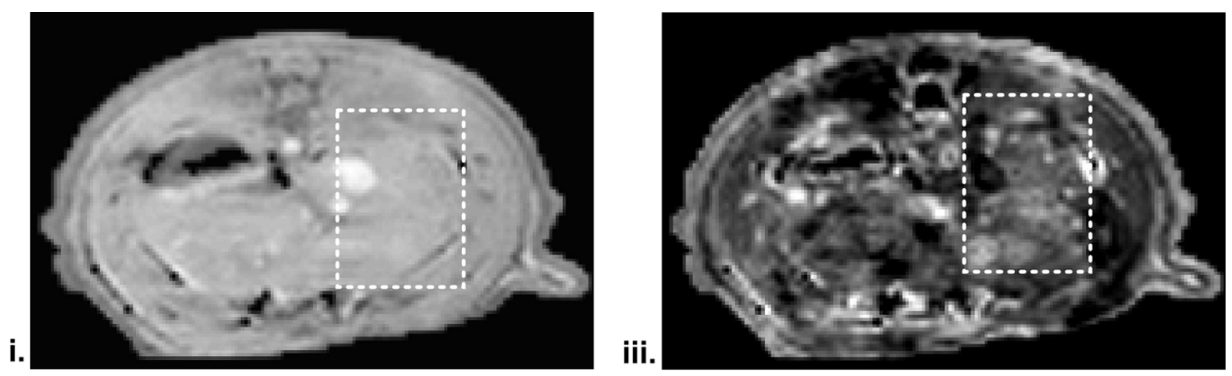

a ii.
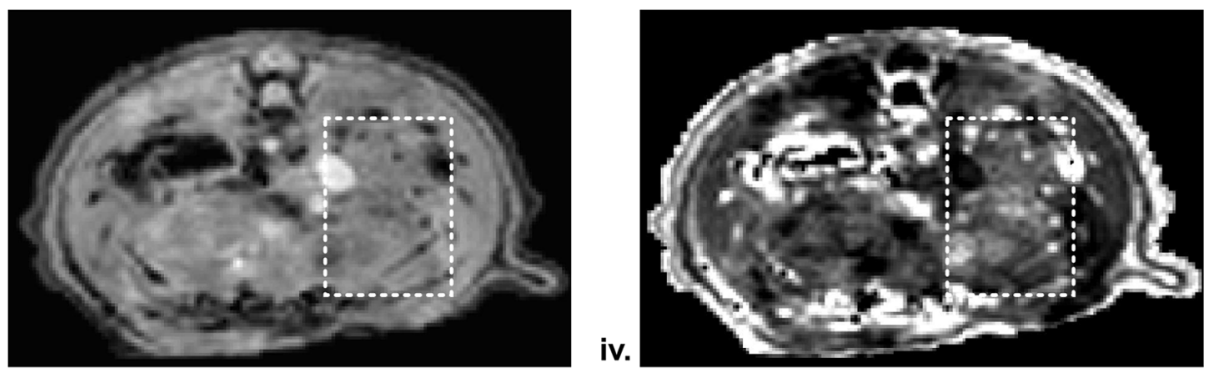

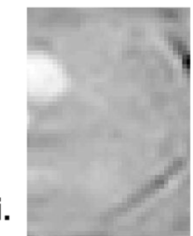

iii.

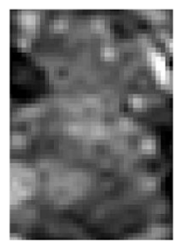

b ii.

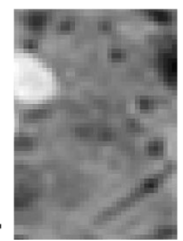

iv.

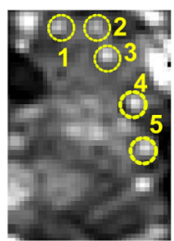

Fig. 2 MR images from a rat that has undergone transplantation of PIs: a axial sample images from 3D variable TE SPGR images from a clinical 3T MR scanner: (i) sub-millisecond first echo image ( $\mathrm{TE}=0.8 \mathrm{~ms}$ ), (ii) second echo image $(\mathrm{TE}=6.75 \mathrm{~ms}$ ), (iii) image difference, (iv) relative subtraction image, $\mathbf{b}$ details from all four

\section{Discussion}

In this study, the development of a sequence that provides sub-millisecond echo times similar to UTE techniques allowed positive contrast imaging of species with very short $\mathrm{T}_{2} *$. As a basis for the development of the sequence, a 3D SPGR template was used, since in addition to high contrast anatomical images, it offers larger volume coverage, and is suitable for application in a breath-hold (i.e., we suppose there will be a benefit for patients who have trouble holding their breath, especially in end-expiration mode). The sequence demonstrated high sensitivity, as even single islets could be visualized in vitro with positive contrast images. Labeled PIs were also identified positively in the rat, as well as in the patient scans.

In addition, the fact that the sequence is fast and flexible could also offer the possibility of adding special

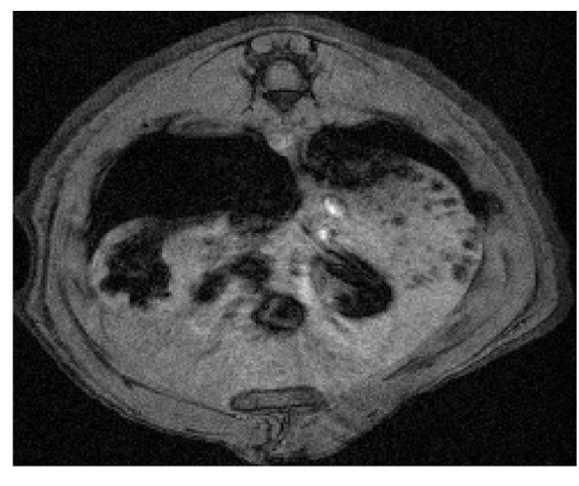

images of sub-Fig. 2a, where the labeled islets are more clearly visible (on sub-Fig. 2biv, the ROIs that were used for CNR calculations are indicated) c high-resolution (i.e. $0.23 \mathrm{~mm}$ ) axial SPGR sample image (from a 2D multi-slice data set) acquired on an experimental $4.7 \mathrm{~T}$ animal scanner

preparation schemes for fat suppression or other utilities. This could be especially useful in the case of obese patients or patients with fibrotic liver.

The technique has shown low sensitivity to shimming issues. During the experiment, only the standard product pre-scan 3D shimming procedure was used. As we can see from Fig. 1, this can be an issue at the edges of phantom, but nevertheless, we see on Fig. 3 that the image quality on the liver does not suffer from any shimming issues.

The background level has lower intensity in the positive contrast images, but is more suppressed in the relative subtraction ones. In this case, the patient was scanned 6 months after transplantation, and the labeling effect is not so strong any more. As a result, thresholding would be challenging, but in the case of patients scanned in a shorter time after the transplantation, it would be probably feasible. 

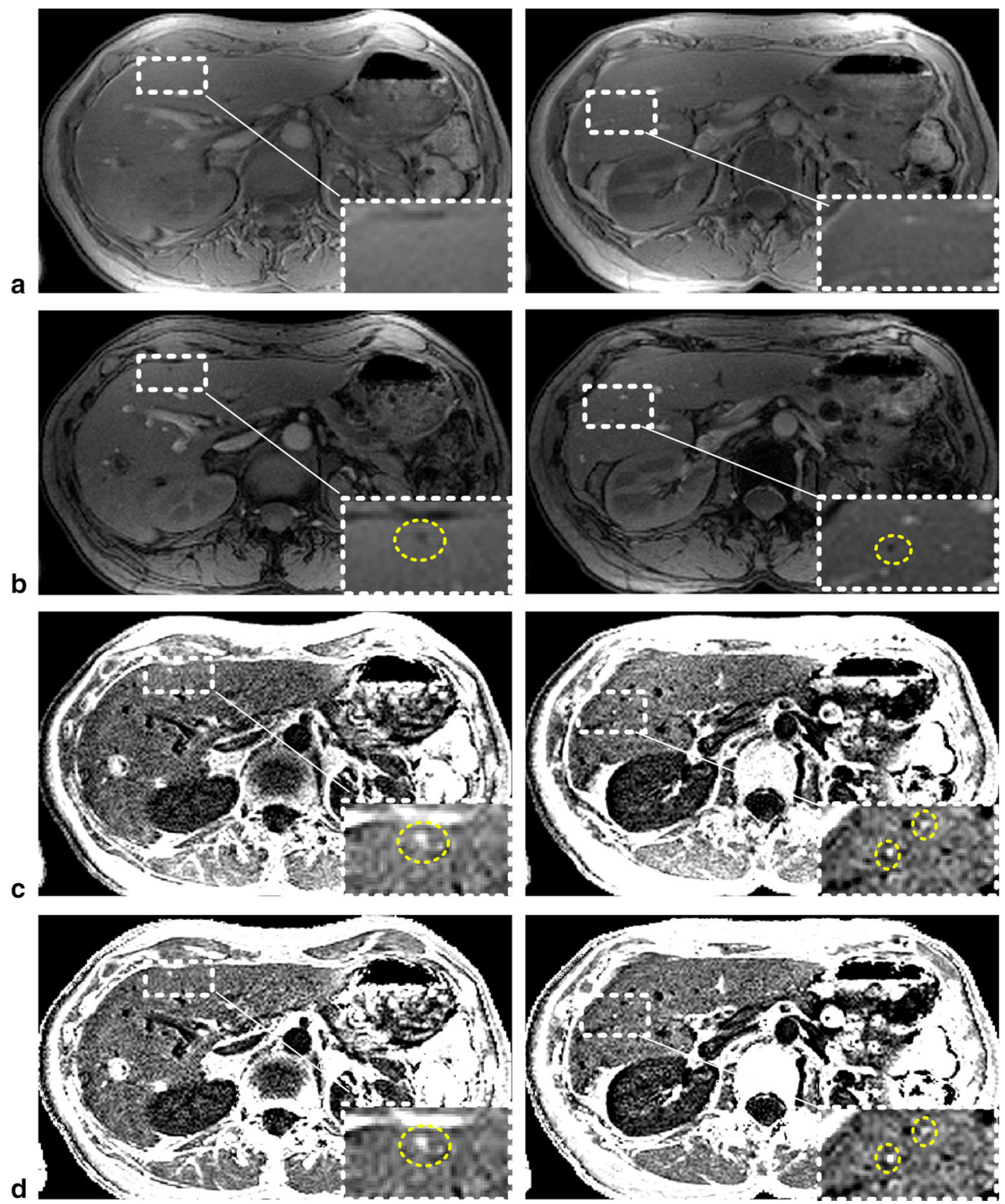

Fig. 3 MR images $\left(1.3 \times 1.3 \times 2.5 \mathrm{~mm}^{3}\right.$, acquisition time $\left.19 \mathrm{~s}\right)$ acquired at two different slice positions (left and right) at 3T with a variable TE 3D SPGR sequences from a patient, who had undergone transplantation of labeled pancreatic islets: a sub-millisecond first

Currently, due to a pause in production of Resovist for human use, further research related to SPIO labeled islets on humans must be delayed. There is active research towards new agents that investigate the use of different particles such as magnetoliposomes [26]. Methods using particles with an iron concentration comparable to the currently used or higher could profit from the proposed method.

A significant finding of this study was the confirmation of the clearance of the area representing transplanted islets in human liver over time by a different imaging approach. It is another part of the mosaic where we try to image transplanted pancreatic islets, because it confirms the echo acquired at $0.65 \mathrm{~ms}$, b second echo at $6 \mathrm{~ms}$, c difference image, d relative subtraction image. The ROIs of the islets that were used for CNR calculations are indicated on Fig. $3 b$

results obtained from previously used animal models and human studies [11]. In addition, the fact that islets could be detected with a double contrast approach in breath-hold scans even after 6 months after transplantation is a promising result for future applications.

The two different positive contrast methods exhibited better contrast than the late echo images. The relative subtraction images yielded comparable, or in some cases, better contrast than the simple subtraction images. In the animal experiment, CNR for islet detection is, in contrast to Crowe et al. [19], comparable in the late echo and the positive contrast image, but lower in the relative contrast image. 
We showed that the use of the proposed 3D vTE SPGR sequence is transferable to the situation in patients with transplanted pancreatic islets, and we believe this approach might be extended to any kind of iron labeled cells. Finally, the most important result from the current study is that achieving sub-millisecond echo time with the proposed 3D short echo time sequence, provides high CNR, good anatomy information and sensitivity to SPIO labeled cells during a breath-hold of a patient.

\section{Conclusion}

The proposed 3D vTE SPGR sequence is sensitive to iron labeled single islets in vitro, and can be used to identify SPIO labeled islets in the liver. The achievement of sub-millisecond echo time while preserving high image contrast allowed successful reconstruction of positive contrast images and relative subtraction images. Islets were identified positively in positive contrast images in an animal in vivo, as well as in a patient. To our knowledge these are the first patient results of positive contrast liver MRI for detecting pancreatic islets. This sequence could improve the segmentation accuracy in unclear cases of islet detection, and relative subtraction could offer important information for threshold identification with increased contrast-to-noise.

Acknowledgments The study was supported by the grant project (Ministry of Health, Czech Republic) for development of research organization 00023001 (IKEM, Prague, Czech Republic)—Institutional support and by grant ENCITE-Seventh EU Framework Program, number 201842 and by the Ministry of education, youth and sports of the Czech Republic, IC 7E08102 and by the grant IGA NT/ 13099 from Ministry of Health of the Czech Republic. This work was supported by Bayer Schering Pharma, of Switzerland. The study sponsor played no role in matters of design, collection, analysis, interpretation of data, and writing of the report. We thank Nicolin Hainc for reading and commenting on the paper.

\section{References}

1. Liu W, Frank JA (2009) Detection and quantification of magnetically labeled cells by cellular MRI. Eur J Radiol 70(2):258-264

2. Langley J, Liu W, Jordan EK, Frank JA, Zhao Q (2011) Quantification of SPIO nanoparticles in vivo using the finite perturber method. Magn Reson Med 65(5):1461-1469

3. Bulte JWM, Kraitchman DL (2004) Iron oxide MR contrast agents for molecular and cellular imaging. NMR Biomed 17(7):484-499

4. Robertson RP (2004) Islet transplantation as a treatment for diabetes-a work in progress. N Engl J Med 350(7):694-705

5. Shapiro AMJ, Lakey JRT, Ryan EA, Korbutt GS, Toth E, Warnock GL, Kneteman NM, Rajotte RV (2000) Islet transplantation in seven patients with Type 1 diabetes mellitus using a glucocorticoid-free immunosuppressive regimen. $\mathrm{N}$ Engl J Med 343(4):230-238
6. Jirák D, Kríz J, Herynek V, Andersson B, Girman P, Burian M, Saudek F, Hájek M (2004) MRI of transplanted pancreatic islets. Magn Reson Med 52:1228-1233

7. Jirak D, Kriz J, Strzelecki M, Yang J, Hasilo C, White DJ, Foster PJ (2009) Monitoring the survival of islet transplants by MRI using a novel technique for their automated detection and quantification. Magn Reson Mater Phy 22(4):257-265

8. Jiao Y, Peng Z-H, Xing T-H, Qin J, Zhong C-P (2008) Assessment of islet graft survival using a 3.0-Tesla magnetic resonance scanner. Anat Rec 291(12):1684-1692

9. Evgenov NV, Medarova Z, Pratt J, Pantazopoulos P, Leyting S, Bonner-Weir S, Moore A (2006) In vivo imaging of immune rejection in transplanted pancreatic islets. Diabetes 55(9):24192428

10. Toso C, Vallee J-P, Morel P, Ris F, Demuylder-Mischler S, Lepetit-Coiffe M, Marangon N, Saudek F, Shapiro AMJ, Bosco D, Berney T (2008) Clinical magnetic resonance imaging of pancreatic islet grafts after iron nanoparticle labeling. Am J Transplant 8(3):701-706

11. Saudek F, Jirak D, Girman P, Herynek V, Dezortova M, Kriz J, Peregrin J, Berkova Z, Zacharova K, Hajek M (2010) Magnetic resonance imaging of pancreatic islets transplanted into the liver in humans. Transplantation 90(12):1602-1606

12. Zacharova K, Berkova Z, Jirak D, Herynek V, Vancova M, Dovolilova E, Saudek F (2012) Processing of superparamagnetic iron contrast agent ferucarbotran in transplanted pancreatic islets. Contrast Media Mol Imaging 7(6):485-493

13. Marzola P, Longoni B, Szilagyi E, Merigo F, Nicolato E, Fiorini S, Paoli GT, Benati D, Mosca F, Sbarbati A (2009) In vivo visualization of transplanted pancreatic islets by MRI: comparison between in vivo, histological and electron microscopy findings. Contrast Media Mol Imaging 4(3):135-142

14. Wu S, Zhang L, Zhong J, Zhang Z (2010) Dual contrast magnetic resonance imaging tracking of iron-labeled cells in vivo. Cytotherapy 12(7):859-869

15. Brisset J-C, Sigovan M, Chauveau F, Riou A, Devillard E, Desestret V, Touret M, Nataf S, Honnorat J, Canet-Soulas E, Nighoghossian N, Berthezene Y, Wiart M (2011) Quantification of iron-labeled cells with positive contrast in mouse brains. Mol Imaging Biol 13(4):672-678

16. Cunningham $\mathrm{CH}$, Arai T, Yang PC, McConnell MV, Pauly JM, Conolly SM (2005) Positive contrast magnetic resonance imaging of cells labeled with magnetic nanoparticles. Magn Reson Med 53(5):999-1005

17. Eibofner F, Steidle G, Kehlbach R, Bantleon R, Schick F (2010) Positive contrast imaging of iron oxide nanoparticles with susceptibility-weighted imaging. Magn Reson Med 64(4):10271038

18. Zhao Q, Langley J, Lee S, Liu W (2011) Positive contrast technique for the detection and quantification of superparamagnetic iron oxide nanoparticles in MRI. NMR Biomed 24(5):464-472

19. Crowe LA, Ris F, Nielles-Vallespin S, Speier P, Masson S, Armanet M, Morel P, Toso C, Bosco D, Berney T, Vallee J-P (2011) A novel method for quantitative monitoring of transplanted islets of langerhans by positive contrast magnetic resonance imaging. Am J Transpl 11(6):1158-1168

20. Bergin CJ, Pauly JM, Macovski A (1991) Lung parenchyma: projection reconstruction MR imaging. Radiology 179(3):777781

21. Robson MD, Gatehouse PD, Bydder M, Bydder GM (2003) Magnetic resonance: an introduction to ultrashort TE (UTE) imaging. J Comput Assist Tomogr 27(6):825-846

22. Martirosian P, Schraml C, Springer F, Schwenzer NF, Würslin C, Schick F and Deimling M (2013), Positive contrast MR imaging of tendons, ligaments, and menisci by subtraction of signals from 
a double echo steady state sequence (Sub-DESS). Magn Reson Med. doi:10.1002/mrm.24651. [Epub ahead of print]

23. Deligianni X, Bär P, Scheffler K, Trattnig S, Bieri O (2012) Highresolution fourier-encoded sub-millisecond echo time musculoskeletal imaging at 3 Tesla and 7 Tesla. Magn Reson Med. doi:10.1002/mrm.24578. [Epub ahead of print]

24. McGibney G, Smith MR, Nichols ST, Crawley A (1993) Quantitative evaluation of several partial Fourier reconstruction algorithms used in MRI. Magn Reson Med 30(1):51-59
25. Santini F, Patil S, Scheffler K (2011) IceLuva: a scripting framework for MR image reconstruction based on free software. Concepts Magn Reson B Magn Reson Eng 39B(1):1-10

26. Ketkar-Atre A, Louchami K, Yin T, Struys T, Malaisse W, Himmelreich U (2013) Longitudinal follow up of pancreatic islets by MRI using labeling with magnetoliposomes. Proc Intl Soc Magn Reson Med 21:4059 